\title{
El relato de los movimientos sociales: claves del discurso ideológico y evolución en los mensajes de 'Democracia Real Ya' (2011-2013)
}

\author{
Celia Berná Sicilia \\ Universidad Católica San Antonio de Murcia \\ cberna@ucam.edu \\ Helena MARTÍNEZ MARTÍNEZ \\ Universidad Católica San Antonio de Murcia \\ hmmartinez@ucam.edu \\ Rocío ZAMORA MEDINA \\ Universidad de Murcia \\ rzamoramedina@um.es
}

\begin{abstract}
Resumen
Este trabajo muestra el perfil ideológico de los mensajes oficiales del movimiento de los indignados que surgió en España en 2011. Para ello, analiza el discurso ideológico de los llamamientos promulgados por Democracia Real Ya (DRY), y se fija en cómo este se autodefine y la manera en que representa a sus enemigos u oponentes. El objetivo es determinar cómo los llamamientos difundidos se configuraron como discursos de carácter ideológico. Los resultados demuestran que estas convocatorias siguieron la estrategia discursiva clásica de los textos ideológicos que opone la autorepresentación positiva de los miembros del ingroup a la presentación negativa de los integrantes del outgroup. Además, el análisis pormenorizado del léxico empleado revela que DRY no lanzó un mensaje unitario a la ciudadanía.
\end{abstract}

\section{The story of social movements: keys in ideological discourse and evolution of the messages from 'Democracia Real Ya' (2011-2013)}

\begin{abstract}
:
This work shows the ideological profile of autorepresentation messages of the indignados movement that emerged in Spain in 2011. It analyzes the ideological discourse of the calls lanched by Democracia Real Ya (DRY), and focuses on how it defines itself and how it represents its enemies or opponents. The aim is to determine how widespread calls were configured as ideological discourses. Results show that these calls followed the classical discursive strategy by opposing ideological texts of positive self-ingroup members to negative presentations of outgroup members. In addition, a detailed lexicon autorepresentation reveals that DRY did not launch a united message to the citizenship.
\end{abstract}

\section{Referencia normalizada:}

Berná Sicilia, C.; Martínez Martínez, H.; Zamora Medina, R. (2013) El relato de los movimientos sociales: claves del discurso ideológico y evolución en los mensajes de 'Democracia Real Ya' (2011-2013). Historia y Comunicación Social. Vol. 18 No Especial Noviembre. Págs. 399-417.

Sumario: 1. Introducción. 2. La importancia del factor identidad en el estudio de los nuevos movimientos sociales contemporáneos. 3.metodología. 4. Análisis y resultados. 5. Conclusiones. 6. Bibliografía. 


\section{Introducción ${ }^{1}$}

El movimiento 15M comenzó con una manifestación puntual el 15 de mayo de 2011 (una semana antes de las elecciones regionales el 22 de mayo en España) que tuvo lugar en varias ciudades españolas y que dio origen a diversas acampadas dispersas por las principales plazas de las ciudades de España. Lo que en principio surgió como un acto de protesta espontáneo y muestra de la indignación de la población por las consecuencias de la crisis económica ${ }^{2}$ y sus repercusiones sobre la tasa de desempleo $^{3}$, el sistema financiero y la ley hipotecaria, así como el escepticismo público hacia los políticos ${ }^{4}$, abarcó a miles de personas de diferente raza, edad y nivel cultural.

El movimiento $15 \mathrm{M}$ se convirtió, de este modo, en un movimiento intergeneracional, en el que el nexo y punto de encuentro entre los diferentes actores que en las redes y en las calles se congregaba era únicamente el sentimiento de indignación. En poco tiempo, la protesta española se extendió a cientos de ciudades y más de ochenta países de todo el mundo con el nombre de "Occupy the Street" (Castells, 2012). Pronto sirvieron de referencia para otros movimientos sociales del contexto internacional (Morell, 2012; Zamora y García, 2013; Perugorría y Tejerina, 2013; Peña-López, Congosto y Aragón, 2013).

De este modo, se fue configurando el 15M como un movimiento social horizontal que presentaba un carácter polifónico debido al gran número de plataformas y agentes sociales que se integraban y participaban en él y que, precisamente porque compartían la indignación ante la situación $\mathrm{d} E l$ País, pronto serían reconocidos popularmente con el nombre de los indignados. Así, el movimiento 15M creció no solo en número, sino en perfiles e identidades que lo formaban.

La creación de asambleas fue una herramienta útil e innovadora para la organización, logística, optimización y desarrollo de recursos de los integrantes del movimiento, pero la organización interna en pequeños grupos derivó también en algunos casos en divisiones: cada pequeño grupo, se organizaba y gestionaba sus recursos, pero también construía su propia identidad, poniendo el foco de atención en seleccionadas temáticas, proponiendo y asistiendo a diferentes actos, etc.

En este sentido, la heterogeneidad de movimiento puede haber sido causa también de la diversificación de los mensajes del movimiento, la incapacidad para transmitir un discurso claro y conciso y fácilmente identificable por parte de la sociedad española, además de ser quizás la base que ha actuado como motivo de la escisión del movimiento y su división en pequeños grupos distintos con intereses, propuestas, motivaciones e identidades propias. Estas escisiones trajeron consigo el despegue de grupos centrados en temas muy específicos, con propuesta, simbología, discurso y, al final, una identidad propia y definida.

Dos años después de que se produjeran las primeras manifestaciones y una vez consolidado y reconocida su influencia a nivel nacional e internacional, el movimiento $15 \mathrm{M}$ ha evolucionado $\mathrm{y}$, con ello, también su discurso. 
Este trabajo pretende mostrar el perfil ideológico de los mensajes oficiales del movimiento, así como los cambios y la evolución que estos han ido sufriendo a lo largo del tiempo, desde un una perspectiva eminentemente semántica, estableciendo una comparación entre los tres discursos promulgados por Democracia Real $\mathrm{Ya}$ (DRY) antes de las grandes manifestaciones convocadas por la plataforma: el primero en las fechas previas al $15 \mathrm{M}$ y los siguientes en los días que precedieron a sus dos aniversarios $-12 \mathrm{M}$ en 2012 y $12 \mathrm{M}$ en 2013 , respectivamente-.

Atendiendo fundamentalmente al modo en que el movimiento se autodefine y a la manera en que representa discursivamente a sus enemigos $u$ oponentes, el objetivo es determinar cómo los llamamientos a la ciudadanía difundidos por DRY se configuraron como discursos de carácter marcadamente ideológico, así como señalar las eventuales alteraciones que con el paso del tiempo se han ido produciendo en el mensaje oficial. El análisis se completará, además, con el examen sistemático de las unidades léxicas a través del software de minería textual Leximancer. Esta aproximación nos permitirá conocer con mayor detalle cuál es la organización semántica que presenta cada una de las convocatorias y dilucidar dónde puso el foco temático la plataforma al llamar a la movilización a la ciudadanía en los años 2011, 2012 y 2013, respectivamente.

La metodología utilizada es de carácter cualitativo, pues se emplean técnicas de análisis del discurso ideológico (Van Dijk, 1996, 2005, 2008) que permiten un acercamiento a las formas de comunicación bajo las que se descubre la ideología que se oculta tras el lenguaje. La estrategia metodológica seguida en el estudio sistemático del léxico empleado en las convocatorias, es, asimismo, de naturaleza cualitativa, aunque se respalda sobre los datos cuantitativos ofrecidos por la herramienta Leximancer.

Los resultados apuntan a que las convocatorias de DRY se han construido siguiendo la estrategia discursiva clásica de los textos ideológicos. Así, los mensajes aplican un esquema polarizado en el que se opone la autorepresentación positiva de los miembros del ingroup (en este caso, DRY) a la presentación negativa de los integrantes del outgroup (en este caso, los poderes políticos y financieros, considerados enemigos de la plataforma). Por otro lado, el estudio pormenorizado del léxico empleado revela que DRY no lanzó un mensaje unitario a la ciudadanía: cada convocatoria se articuló con una estructura semántica distinta y con una organización temática divergente. Además, la información que se desprende del análisis de los elementos léxicos que configuran el discurso de la plataforma desvela que el mensaje dirigido a los ciudadanos parece haber ido adquiriendo, con el tiempo, matices más beligerantes e ideológicos. 


\section{La importancia del factor identidad en el estudio de los nuevos movimientos sociales contemporáneos}

Superada la fase de crecimiento y desarrollo de la sociedad industrial y en un tiempo en el que conviven subculturas con marcado carácter identitario y una sociedad altamente tecnológica, bajo el marco de la globalización, surgen nuevos movimientos sociales que han resultado difícilmente catalogables y analizables desde las teorías y perspectivas tradicionales (Diani, 1992).

El estudio de los movimientos sociales en términos de conflictos de clase que caracterizó las corrientes sociológicas estructural-funcionalista y la tradición marxista, evidenció la necesidad de nuevas perspectivas y recursos para hacer frente al análisis de las nuevas movilizaciones ciudadanas.

A partir de los años sesenta, las perspectivas teóricas tradicionales evolucionaron hacia cuatro enfoques predominantes en el estudio de movimientos sociales, con diferencias en sus visiones y focos de atención. Así, la teoría del comportamiento colectivo entendió los fenómenos colectivos como una actividad que apunta a la producción de nuevas normas y solidaridades (Diani, 1992). En contra, se posicionó la teoría de movilización de recursos, la cual entiende la movilización colectiva como acción racional, con objetivos determinados y con dependencia de los recursos organizativos (Laraña,1999). La teoría de la oportunidad política hizo hincapié en la relación entre las movilizaciones colectivas y las instituciones políticas, especialmente en el papel que cumple el Estado. En palabras de Tarrow, se trata de "[...] dimensiones consistentes (aunque no necesariamente formales, permanentes o nacionales) del entorno político, que fomentan o desintegran la acción colectiva entre la gente [...]" (Tarrow, 1996). Por último, la teoría de los nuevos movimientos sociales, entiendía el movimiento social como "la conducta colectiva organizada de un actor luchando contra su adversario por la dirección social de la historicidad en una colectividad concreta (Touraine, 2006).

A pesar de la novedad que representan estas aproximaciones, resultan insuficientes para definir las nuevas movilizaciones colectivas contemporáneas, caracterizadas por la importancia que adquiere el concepto de identidad, por un lado, así como por su enorme capacidad de autocomunicación (Castells, 2007) en el nuevo ecosistema mediático.

Otro rasgo claramente definitorio de estos nuevos movimientos sociales tiene que ver con su enorme capacidad de comunicación (que crearon sus propios medios de comunicación alternativos), sin líderes, y con una estructura comunicativa descentralizada y menos jerárquica que los movimientos sociales previos, donde el uso de las tecnologías digitales es esencial para el surgimiento de las propiedades de resistencia (RP) (Atkinson, 2010).

En este sentido, el 15M se caracterizó por mostrar múltiples identidades, en parte aprovechando los recursos que ofrecían las redes sociales y las estructuras horizontales. Un detalle importante que ilustra la influencia de las redes sociales en el 
movimiento es que sólo dos de sus páginas en Facebook reunió a más de 600.000 usuarios. La llamada autocomunicación de masas (Castells, 2007) o comunicación digital abierta, personalizada y masiva, ofreció un medio extraordinario para este movimiento, con el fin de construir su autonomía y hacer frente a las instituciones de la sociedad en sus propios términos.

\title{
3. Metodología
}

El análisis de los mensajes de Democracia Real Ya con el que se pretende mostrar el perfil ideológico de las convocatorias, así como la evolución de temas y asuntos abordados se efectuará a través de distintas vertientes metodológicas. De un lado, se aplicará una metodología de carácter cualitativo en la que se atenderá a la representación que en cada texto presentan las categorías definitorias de las ideologías: identidad/membresía, actividades, metas, normas y valores y recursos (Van Dijk, 1996, $2005,2008)$. De otro, se procederá a examinar el material textual gracias al software de minería textual Leximancer. Esta herramienta nos facilitará la aprehensión de la estructura semántica y de la articulación temática de cada uno de los textos, pues permite extraer los conceptos clave de un determinado texto, en virtud de los índices de frecuencia y utorepresen que las voces que lo componen presentan en él, proporcionando un mapa conceptual donde se representan esos conceptos y la interrelación de los mismos dentro del corpus analizado. Se utilizará, por tanto, en esta segunda parte del análisis, una metodología cualitativa, aunque asentada y sustentada sobre datos cuantitativos.

Es preciso señalar, asimismo, que, en la primera etapa del análisis, comprobaremos que los textos analizados muestran información que responde esencialmente a las siguientes preguntas:

\begin{abstract}
¿Quiénes somos nosotros?, ¿Quiénes (no) pertenecen a nosotros?, ¿Qué hacemos nosotros?, ¿Cuáles son nuestras actividades?, ¿Qué se espera de nosotros?, ¿Cuáles son las metas de estas actividades?, $i$ Qué normas y valores respetamos en tales actividades?, ¿Con qué grupos estamos relacionados: quiénes son nuestros amigos y quiénes nuestros enemigos?, ¿Cuáles son los recursos a los que típicamente tenemos o no acceso (privilegiado)? (Van Dijk, 1996: 28).
\end{abstract}

En este sentido, prestaremos especial atención a las unidades léxicas que se utilizan para describir a los que no pertenecen al grupo -que denominaremos "enemigos"- dentro de las convocatorias porque son las que más pistas ofrecerán sobre el posicionamiento ideológico de los mensajes lanzados por esta plataforma para movilizar a los ciudadanos. Tal como se ha demostrado desde distintos ámbitos de estudio como la teoría de intergrupos, las teorías del estereotipo y las investigaciones sobre las cogniciones sociales, la ideología y las representaciones mentales subyacentes de los grupos facilitan la creación de conceptos evaluativos globales que inciden en los mecanismos de selección léxica. 
En los textos ideológicos, el esquema maniqueo que polariza el discurso entre "buenos" y "malos" constituye la estrategia discursiva que suele activarse predominantemente. Así, en este tipo de mensajes los miembros del grupo o sus aliados aparecen representados habitualmente de forma positiva, mientras que sus oponentes figuran en términos eminentemente negativos.

La semántica ideológica subyacente a tal selección léxica sigue una pauta estratégica muy clara, esto es, en general se tiende a describir en términos positivos a los grupos a los que pertenecemos (ingroups) y a sus miembros, así como a sus amigos, aliados o seguidores, mientras que a los grupos ajenos (outgroups), a los enemigos u oponentes se les describe en términos negativos. (Van Dijk, 1996: 23-24).

Con todo, es preciso advertir que las estructuras ideológicas del discurso no solo se dejan entrever a través los adjetivos o los sustantivos usados para describir al gupo al que se pertenece (ingroup) y a los otros grupos (outgrup) y sus atributos, sino que también aparecen representadas a través de otras estructuras más complejas vinculadas con acciones, objetos, lugares, o acontecimientos específicos en los que están implicados los grupos. De este modo, es posible analizar los cuadros de descripción positiva y negativa y los cuadros atribuciones de acciones positivas o negativas.

Para recabar los datos del análisis, aplicaremos la una tabla metodológica a cada uno de los textos (Convocatoria mayo 2011: texto 1; Convocatoria mayo 2012: texto 2; Convocatoria mayo 2013: texto 3). Esta ficha de análisis se asienta sobre los planteamientos teóricos que hemos señalado anteriormente e incide en los aspectos que configuran el discurso de la plataforma DRY como un discurso marcadamente ideológico (identidad, actividades, metas, normas y valores y recursos).

\section{Análisis y resultados}

A continuación, desvelamos cuáles son los resultados derivados de la aplicación de la ficha de análisis del discurso que hemos diseñado y aplicado a las convocatorias lanzadas por DRY en 2011, 2012 y 2013, respectivamente. Asimismo, ofrecemos la información extraída del análisis realizado a través del software Leximancer.

\subsection{Análisis del perfil ideológico de las convocatorias}

De acuerdo con la información que se desprende de los textos 1, 2 y 3, el ingroup se identifica con un colectivo de personas muy amplio: la ciudadanía en general. De hecho, para incidir en el carácter inclusivo de los mensajes, se usa de forma reiterada la primera persona del plural (nosotros) a lo largo de todas las convocatorias.

Sin embargo, desde el primer mensaje, los miembros de la plataforma DRY marcan también las distancias con los integrantes del outgroup. De la gran mayoría ciudadana que integraría el ingroup, hay que excluir a las personas que ostentan los poderes políticos y financieros, pues estos dos colectivos emergen en los mensajes como 
los dos grandes grupos de oponentes. Explícitamente, los enemigos que se nombran en los textos son los mercados, las corporaciones bancarias, los dirigentes políticos y financieros y la clase política en general. No hay, por tanto, una personalización acusada en la configuración del perfil antagónico del ingroup: se esbozan enemigos sin rostro y de corte genérico. Con todo, en el tercer texto hay una mayor concreción en la delimitación de un enemigo al remitir de modo concreto a "la troika", pues aunque esta entidad también se caracteriza por cierta indefinición, se vincula con tres conocidas instituciones (Comisión Europea, Fondo Monetario Internacional y Banco Central Europeo), y al frente de ellas hay mandatarios también conocidos por la ciudadanía. Por otro lado, hay que subrayar, asimismo, que en el tercer texto entra otro difuso enemigo en escena: el sistema corrupto. Es solo en esta última convocatoria cuando DRY muestra explícitamente su oposición al sistema establecido.

En la descripción del ingroup, es preciso señalar que DRY se autodefine como una plataforma inclusiva integrada por individuos pasivos exentos de culpa dentro de la sociedad: son "víctimas y testigos" de la situación actual que atravesamos (Texto 1). En la misma línea se sitúa el movimiento al identificar a los miembros del ingroup en el texto 2 como "condenados a la pobreza, a la incertidumbre y a los riesgos medioambientales". La construcción enfatizada de este perfil victimista del ingroup contrasta con la indefinición del outgroup, pues este aparece mayoritariamente retratado en los textos en virtud de las acciones (negativas) que se le atribuyen: "Nos dejan indefensos", "no invierten en el ser humano ni en sus necesidades reales", "desarrollan una gestión que apoya la especulación", "dejan a gente sin trabajo, a mayores abandonados, a madres y padres sin derechos, a gente en la calle", "someten a un genocidio financiero a la población", "aplican recortes al bienestar de los ciudadanos", "contraen deudas", etc.). En este sentido, es reseñable destacar que hay pocos adjetivos o sustantivos que describan a los antagonistas, aunque en la tercera convocatoria sí hay algunos que reflejan una imagen muy negativa de ellos: son corruptos, codiciosos, interesados, manipuladores, partidistas y cometen desmanes contra la ciudadanía.

La atribución de acciones negativas al outgroup se opone, a su vez, a la presentación de las acciones positivas desarrolladas por el ingroup (reivindican nuestros derechos, ejercitan la responsabilidad democrática, exigen transparencia, responsabilidad, que se trabaje en pro del bien común, etc.). Con todo, es preciso advertir que se trata de actividades de carácter genérico. No es hasta el texto 3 cuando estas actividades abandonan la categoría de lo "etéreo" y adquieren mayor concreción. Una vez que la plataforma ha consolidado su andadura como movimiento social, el inventario de acciones positivas asignadas al ingroup aumenta considerablemente. Aunque sigue habiendo referencias a acciones poco concretas, la tercera convocatoria se convierte en un pequeño muestrario de lo realizado y conseguido hasta el momento (eventos, manifestaciones y concentraciones de protesta, ILP de los desahucios, etc.) y de lo que está por venir (acción: escrache al sistema).

Para llevar todas estas tareas a cabo, DRY dice contar solo con dos poderosos recursos que hace suyos: la unión de toda la ciudadanía y el espacio público (la calle). 
Asimismo, a partir de la segunda convocatoria, se hace mención de otro recurso al alcance del ingroup: son muchos (ciudadanía) frente a pocos (poderes políticos y financieros). Finalmente, conviene advertir, además, que todas las acciones del ingroup se inspiran y proyectan desde un conjunto de normas y valores positivos que constituyen las señas de identidad del movimiento, a saber: humanismo, apelación a los sentimientos y la condición humana y carácter asindical y apolítico de las reivindicaciones. Estos pilares morales y sociales son los que condicionan las metas y fines que persigue el ingroup y que poseen también un carácter muy positivo: la defensa de los derechos de los ciudadanos, la búsqueda de la justicia, la equidad social y el bien común y la construcción de otro estado español, si bien en el tercer texto nuevamente se da un paso más allá.

Otros aspectos relevantes que se derivan del examen de los datos extraídos tienen que ver con la aplicación de la estrategia de polarización discursiva en las tres convocatorias, propia de los discursos ideológicos. En los tres textos encontramos el esquema "Nosotros (muchos y buenos) contra Ellos (pocos y malos)" y en él la balanza se inclina con claro favoritismo hacia el ingroup.

La autorepresentación positiva del ingroup se ve reforzada por: la inclusión de un perfil victimista en la identificación de sus integrantes, la atribución y asignación de acciones positivas, la persecución de fines o metas positivos y la apelación a valores y normas que generan una simpatía generalizada. Por el contrario, la negativización del outgroup se materializa discursivamente gracias a recursos como la asignación de rasgos negativos, la atribución de acciones negativas o la vaguedad en la identificación y definición de sus integrantes.

Por último, es reseñable también que parece observarse un mayor grado de animadversión hacia los enemigos y también un mayor grado de crispación y radicalidad en el mensaje del texto 3, lo que se refleja en diferentes aspectos como el empleo de adjetivos calificativos de carácter muy negativo para describir al outgroup ("manipuladores", corruptos", etc.), la autodefinición de los miembros del grupo como "luchadores" y la oposición explícita del movimiento al sistema establecido.

A continuación, ofrecemos los datos generales extraídos del análisis de los textos (Tablas 1,2 y 3 ). 
Tabla 1. Convocatoria DRY 2011 (Texto 1)

\begin{tabular}{|c|c|c|c|c|c|c|c|}
\hline Identidad & & & & Actividades & Metas & $\begin{array}{l}\text { Normas y } \\
\text { valores }\end{array}$ & Recursos \\
\hline $\begin{array}{l}\text { Ingroup } \\
\text { ciudadanía en } \\
\text { general }\end{array}$ & & $\begin{array}{l}\text { Outgroups } \\
\text { Mercados } \\
\text { Corporaciones } \\
\text { bancarias } \\
\text { Dirigentes } \\
\text { políticos y } \\
\text { financieros } \\
\text { Clase política }\end{array}$ & & $\begin{array}{l}\text { Convocatoria } \\
\text { de una } \\
\text { manifestación }\end{array}$ & $\begin{array}{l}\text { Justicia y } \\
\text { equidad } \\
\text { social } \\
\text { Bien común } \\
\text { otro estado } \\
\text { español } \\
\text { posible y } \\
\text { necesario }\end{array}$ & $\begin{array}{l}\text { Asindical } \\
\text { Apolítico y } \\
\text { apartidista } \\
\text { Humanismo } \\
\text { No hay } \\
\text { banderas (sin } \\
\text { distinción } \\
\text { ideológica, } \\
\text { creencia ni } \\
\text { nacionalidad) } \\
\text { Sentimientos/ } \\
\text { condición } \\
\text { humana } \\
\end{array}$ & $\begin{array}{l}\text { Unión } \\
\text { calle }\end{array}$ \\
\hline Descripción & $\begin{array}{l}\text { Atribución de } \\
\text { acciones }\end{array}$ & Descripción & $\begin{array}{l}\text { Atribución de } \\
\text { acciones }\end{array}$ & & & & \\
\hline \begin{tabular}{|l} 
Pronombre \\
personal \\
de primera \\
persona del \\
plural \\
(nosotros) \\
Testigos \\
Víctimas \\
\end{tabular} & $\begin{array}{l}\text { Salgamos a } \\
\text { la calle por } \\
\text { otro estado } \\
\text { español posible } \\
\text { y necesario. } \\
\text { Reivindiquemos a } \\
\text { la clase política } \\
\text { Ejercicio de } \\
\text { responsabilidad } \\
\text { ciudadana } \\
\text { exigir } \\
\text { transparencia, } \\
\text { responsabilidad, } \\
\text { bien común } \\
\text { reivindicar } \\
\text { nuestros derechos } \\
\text { de forma pacífica } \\
\text { y exigimos que se } \\
\text { trabaje en pro del } \\
\text { bien de todos los } \\
\text { ciudadanos. } \\
\text { hacer grandes } \\
\text { cosas }\end{array}$ & & $\begin{array}{l}\text { Nos dejan } \\
\text { indefensos } \\
\text { No invierten en } \\
\text { el ser humano } \\
\text { ni en sus } \\
\text { necesidades } \\
\text { reales } \\
\text { Desarrollan } \\
\text { una gestión } \\
\text { que apoya la } \\
\text { especulación } \\
\text { gente sin } \\
\text { trabajo, mayores } \\
\text { abandonados, } \\
\text { madres y padres } \\
\text { sin derechos, } \\
\text { gente en la } \\
\text { calle, extinción } \\
\text { paulatina de la } \\
\text { salud pública y } \\
\text { la educación } \\
\text { se venden } \\
\text { y dejan de } \\
\text { trabajar para la } \\
\text { ciudadanía } \\
\text { legislan a favor } \\
\text { de las grandes } \\
\text { corporaciones } \\
\text { bancarias }\end{array}$ & & & & \\
\hline
\end{tabular}

Fuente: elaboración propia. 
Tabla 2. Convocatoria DRY 2012 (Texto 2).

\begin{tabular}{|c|c|c|c|c|c|c|c|}
\hline Identidad & & & & Actividades & Metas & $\begin{array}{l}\text { Normas y } \\
\text { valores }\end{array}$ & Recursos \\
\hline \begin{tabular}{|l|} 
Ingroup \\
ciudadanía en \\
general ( $99 \%$ de \\
la población)
\end{tabular} & & $\begin{array}{l}\text { Outgroups } \\
\text { Políticos y } \\
\text { banqueros }\end{array}$ & & $\begin{array}{l}\text { Convocatoria } \\
\text { de una } \\
\text { manifestación }\end{array}$ & $\begin{array}{l}\text { Reclamar justicia social, } \\
\text { defender los derechos } \\
\text { de la ciudadanía, hacer } \\
\text { que los políticos nos } \\
\text { escuchen. }\end{array}$ & $\begin{array}{l}\text { Justicia } \\
\text { social }\end{array}$ & $\begin{array}{l}\text { Unión, calle } \\
\text { Muchos frente } \\
\text { a pocos. }\end{array}$ \\
\hline Descripción & $\begin{array}{l}\text { Atribución } \\
\text { de acciones }\end{array}$ & Descripción & \begin{tabular}{|l|} 
Atribución de \\
acciones
\end{tabular} & & & & \\
\hline \begin{tabular}{|l|} 
Pronombre \\
personal de \\
primera persona \\
del plural \\
(nosotros) \\
Condenados a \\
la pobreza, a la \\
incertidumbre \\
y a los riesgos \\
medioambientales \\
No somos \\
mercancía
\end{tabular} & $\begin{array}{l}\text { No dan un } \\
\text { paso atrás en } \\
\text { la defensa } \\
\text { de nuestros } \\
\text { derechos }\end{array}$ & & $\begin{array}{l}\text { Se benefician a nuestra } \\
\text { costa } \\
\text { Nos condenan a } \\
\text { la pobreza, a la } \\
\text { incertidumbre } \\
\text { y a los riesgos } \\
\text { medioambientales } \\
\text { No nos escuchan } \\
\text { No hacen valer } \\
\text { nuestras necesidades }\end{array}$ & & & & \\
\hline
\end{tabular}

Fuente: elaboración propia.

Tabla 3. Convocatoria DRY 2013 (Texto 3)

\begin{tabular}{|c|c|c|c|c|c|c|c|}
\hline Identidad & & & & Actividades & Metas & \begin{tabular}{|l|}
$\begin{array}{l}\text { Normas y } \\
\text { valores }\end{array}$ \\
\end{tabular} & Recursos \\
\hline \begin{tabular}{|l} 
Ingroup \\
ciudadanía \\
en general \\
(99\% de la \\
población)
\end{tabular} & & $\begin{array}{l}\text { Outgroups } \\
\text { Políticos y } \\
\text { banqueros }\end{array}$ & & $\begin{array}{l}\text { (Antes) Convocatoria de } \\
\text { eventos, manifestaciones } \\
\text { y concentraciones de } \\
\text { protesta } \\
\text { (Ahora) Acción: } \\
\text { escrache al sistema } \\
\text { Presentación de la ILP } \\
\text { desahucios } \\
\text { Lucha contra las } \\
\text { políticas de la troika }\end{array}$ & \begin{tabular}{|l} 
Justicia y \\
equidad \\
Defensa de \\
derechos \\
Cambio en \\
el sistema: \\
nuevo proceso \\
constituyente \\
Llevar a su \\
final al sistema \\
corrupto \\
\end{tabular} & $\begin{array}{l}\text { Movimiento } \\
\text { de carácter } \\
\text { inclusivo, } \\
\text { apartidista y } \\
\text { asindical. } \\
\text { Única } \\
\text { bandera: } \\
\text { espacio } \\
\text { común de } \\
\text { lucha. }\end{array}$ & $\begin{array}{l}\text { Muchos } \\
\text { frente a } \\
\text { pocos } \\
\text { Calle } \\
\text { Unión }\end{array}$ \\
\hline Descripción & Atribución de acciones & Descripción & \begin{tabular}{|l} 
Atribución de \\
acciones
\end{tabular} & & & & \\
\hline \begin{tabular}{|l|} 
Pronombre \\
personal \\
de primera \\
persona del \\
plural \\
(nosotros) \\
Víctimas \\
Más \\
determinación \\
que nunca \\
Luchadores
\end{tabular} & $\begin{array}{l}\text { Lucha intensa en la calle y } \\
\text { en frente judicial } \\
\text { Diseño de un plan de } \\
\text { rescate ciudadano. } \\
\text { Hacen prevalecer derechos } \\
\text { e intereses de las personas } \\
\text { sobre los de los mercados y } \\
\text { los poderes financieros } \\
\text { se defienden de los } \\
\text { enemigos } \\
\text { Generar la necesaria } \\
\text { voluntaad política para } \\
\text { atender las legítimas } \\
\text { reivindicaciones } \\
\text { ciudadanas. }\end{array}$ & \begin{tabular}{|l|} 
Codiciosos \\
Desmanes \\
Interesados, \\
partidistas \\
Manipuladores \\
corrupto
\end{tabular} & $\begin{array}{l}\text { Contraen deudas } \\
\text { Aplican recortes } \\
\text { al bienestar de } \\
\text { una ciudadanía } \\
\text { Manipulan } \\
\text { interesadamente } \\
\text { a la sociedad }\end{array}$ & & & & \\
\hline
\end{tabular}

Fuente: elaboración propia. 


\subsection{Análisis semántico a través de leximancer}

El análisis del contenido textual de las tres convocatorias de DRY a través de Leximancer nos permite establecer cuáles fueron los temas dominantes, así como advertir las diferencias de corte eminentemente semántico que existen en el discurso que la plataforma lanzó a la ciudadanía. Este software de minería textual proporciona un análisis automático de los textos y facilita la visualización gráfica de la información textual, pues genera mapas conceptuales en los que se señalan los términos más relevantes que componen la muestra y la interrelación que existe entre ellos, al ofrecer datos sobre la autorepresentación de las voces.

Aplicamos Leximancer a los tres textos objeto de análisis y estos son los resultados obtenidos. En el texto 1, tal como muestra la Figura 1, observamos que el mensaje se articula de forma significativa en torno a dos ejes semánticos interconectados: calle y ciudadanos. La herramienta considera que estos dos componentes son los que mayor protagonismo ostentan dentro del texto y los denomina "temas", pero no por ser los más frecuentes, sino por identificarse con las voces que mayor número de asociaciones con otros términos presentan en la muestra. El resto de palabras que se representan a menor tamaño son los que la herramienta denomina "conceptos" (políticos, mayo, común, problemas, etc.). Estos sí son los términos que mayor índice de frecuencia tienen en el corpus textual analizado y, por ello, se consideran, asimismo, relevantes.

Dentro de la esfera "calle", la de mayor extensión en este texto, hallamos palabras vinculadas a la denuncia social que realiza la plataforma y a la finalidad que persiguen las acciones de DRY: reivindicación de derechos, búsqueda del bien común, resolución de los problemas de la sociedad, etc. La urgencia de actuación por parte del cuerpo social también es evidenciada en el texto a través voces como momento y la forma no personal del verbo ocurriendo que imprime la sensación de inmediatez. Asimismo, resulta interesante en esta órbita de significación la relevancia adquirida por la preposición negativa sin, utilizada en esta convocatoria para hacer alusión a las privaciones que los políticos y los banqueros imponen a la población (les dejan $\sin$ derechos, $\sin$ trabajo, etc.) y para expresar el carácter abierto e inclusivo del movimiento (sin banderas, sin ideología). Finalmente, también vemos representados de forma destacada dentro del mapa conceptual algunos términos que se asocian a los rasgos identitarios del movimiento (banderas, ciudadana, forma) y uno de los principales antagonistas del ingroup -los políticos-.

Es preciso advertir, por otro lado, que los conceptos destacados con mayor brillo -políticos, sin, calle- se corresponden con aquellos que, dentro de la muestra, se revelan más frecuentes y que la cercanía o lejanía con la que aparecen representados en el mapa se vincula con los índices de autorepresentación de las voces. Esto es, de acuerdo con los datos, dentro del texto 1, forma y momento se presentan en los contextos de forma simultánea. 
Figura 1. Mapa conceptual texto 1

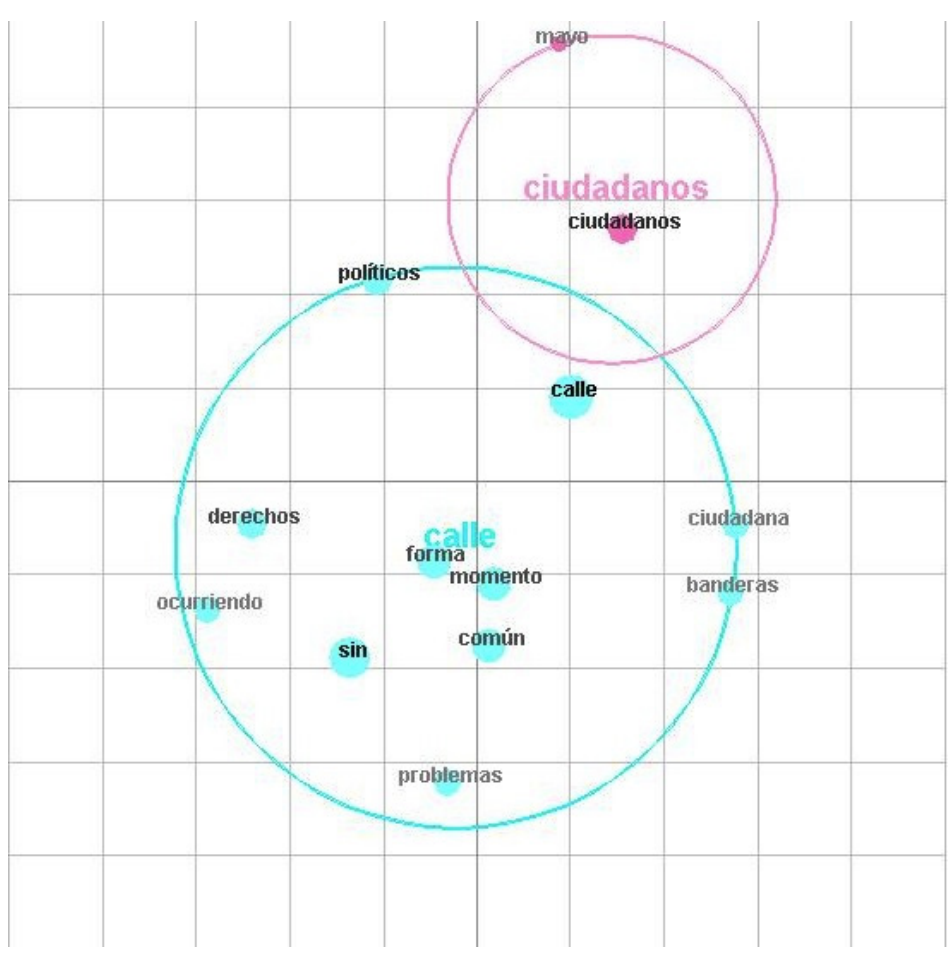

Fuente: elaboración propia.

En el segundo texto, de acuerdo con la Figura 2, el discurso se articula también en torno a dos polos significativos interrelacionados: recursos y diaria. De un lado, tenemos la órbita "diaria" que es la que mayor extensión significativa presenta. En ella, hallamos términos que se vinculan con la denuncia social que quiere realizar la plataforma DRY y favorecen el proceso de victimización del ingroup, integrado por la mayoría de la población mundial. Actualmente, la sociedad se ve ampliamente desfavorecida y está condenada a no tener futuro por una distribución no equitativa de los recursos: son solo unos pocos los que obtienen beneficio y son precisamente ellos quienes de modo irresponsable adoptan riesgos económicos.

La órbita de menor tamaño, "Recursos", se correlaciona, a su vez, con la finalidad de denuncia social que persigue la convocatoria. Así, en ella figuran voces que inciden en la idea de que, debido a la irresponsabilidad de generaciones anteriores (herencia), vivimos en una sociedad "inmensamente pobre" en la que escasean los" recursos". Todo ello justifica, asimismo, la movilización ciudadana y, por ese motivo, aparecen también, en esta esfera significativa, las palabras clave que invitan a la manifestación: calle y mayo. 
Figura 2. Mapa conceptual del texto 2.

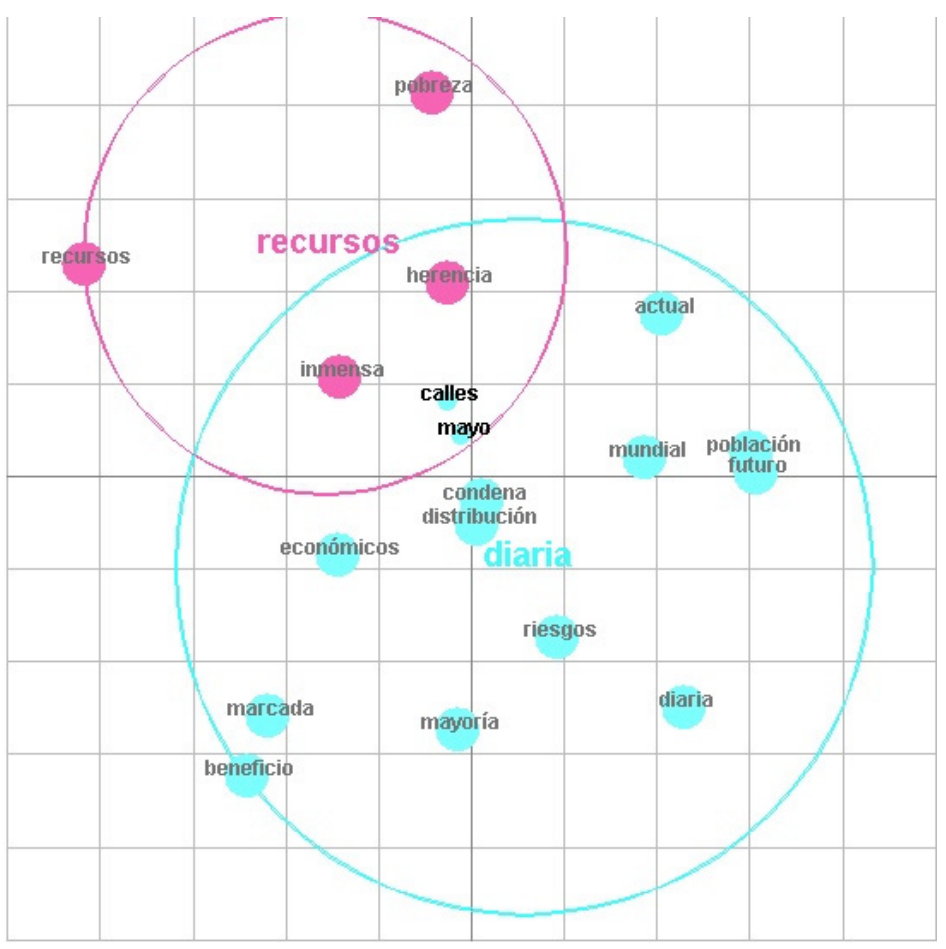

Fuente: elaboración propia.

Por su parte, en la tercera convocatoria de DRY, parece haber mayor atomización semántica. Conforme se desprende de la Figura 3, encontramos cuatro temas destacados: sistema, lucha, genocidio financiero y mercados. Existe, sin embargo, una estrecha interconexión entre todos estos términos, ya que en ellos se condensa el mensaje esencial que desea transmitirse a la ciudadanía a través de esta convocatoria. Así, este tercer texto de DRY se construye genéricamente como una invitación para que la población a luche contra sus enemigos (mercados y sistema) con el objetivo de alcanzar una meta bien delimitada: el genocidio financiero.

Dentro de cada órbita, vemos representados, asimismo, algunos conceptos relevantes vinculados con los temas. La esfera de "Sistema", término que se asocia en el texto 3 significativamente con uno de los enemigos del ingroup, incorpora los términos años y financieros; el tema mercados, con el que se alude a otro de los miembros del outgroup, configura una órbita propia con otros dos conceptos que se refieren a quienes integran ese ente etéreo mercados: poderes (financieros) y personas. Por su parte, en la órbita "Lucha" se incluyen dos voces que concretan cómo se va a desarrollar de modo concreto el combate contra el sistema y los mercados: a través de 
diversas acciones y eventos. Finalmente, la esfera "Genocidio financiero" cuenta con un único integrante que es el propio compuesto sintagmático: genocidio financiero.

Resulta relevante advertir, además, que los términos más destacados por su frecuencia dentro de la muestra correspondiente al texto 3 son lucha y sistema.

Figura 3. Mapa conceptual del texto 3

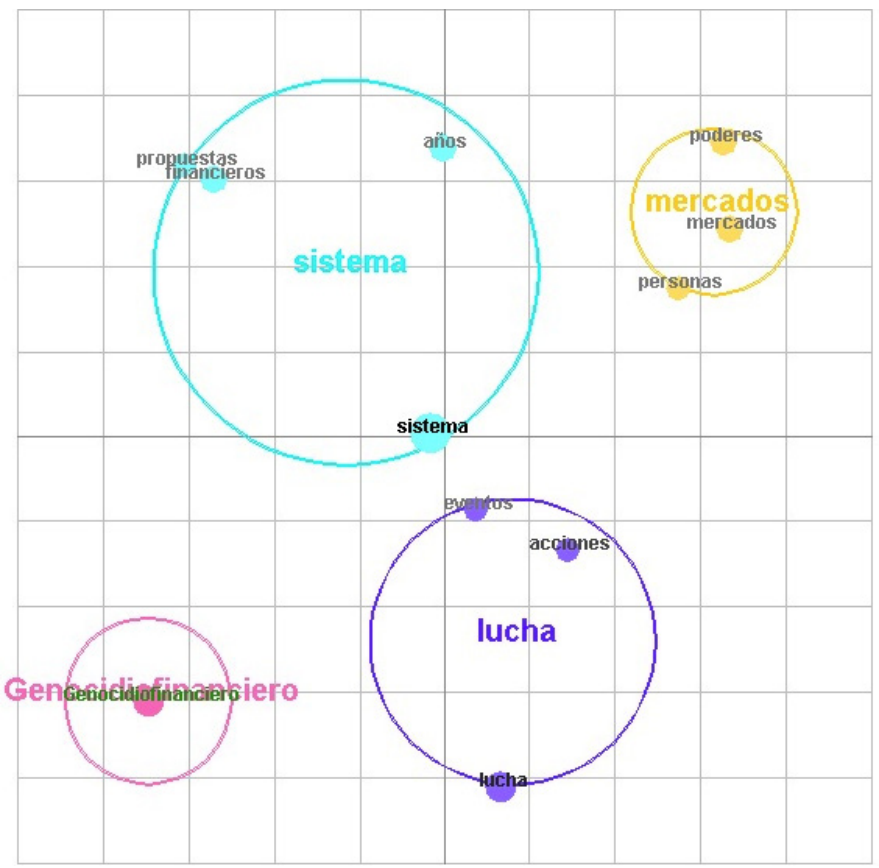

Fuente: elaboración propia.

La comparativa de los resultados obtenidos tras el análisis semántico revela que cada convocatoria se articuló con una estructura semántica distinta y focalizó la atención en aspectos temáticos divergentes. Así, en un primer momento, la plataforma centró su discurso movilizador en la autodefinición del ingroup, marcando distancias con los miembros del outgroup y delimitando, asimismo, las metas y fines perseguidos por el movimiento y sus normas y valores. En el segundo mensaje, se deja relativamente de lado la autodefinición y la alusión a las metas y a los valores de la plataforma, pues la llamada a la movilización ciudadana se orienta más bien hacia la denuncia social: la falta de recursos que sufre la sociedad, provocada por la irresponsabilidad de los dirigentes políticos y financieros, es la que justifica las acciones de protesta.

La última convocatoria es, con todo, la que adquiere tintes más beligerantes. La plataforma adopta un discurso más radical y de enfrentamiento hacia el outgroup, pues, en este mensaje, los términos que mayor extensión e importancia significativa alcanzan son "sistema" y "lucha". 
Finalmente, cabe advertir, asimismo, que, en los dos primeros textos, se observa la presencia de un contenido de índole más política y social frente al contenido más ideológico del tercer mensaje.

\section{Conclusiones}

Dos años después de que se produjeran las primeras manifestaciones y una vez consolidado y reconocida su influencia a nivel nacional e internacional, el movimiento $15 \mathrm{M}$ ha ido evolucionando y, con ello, también su discurso. Este trabajo ha ofrecido interesantes resultados sobre el perfil ideológico de los mensajes oficiales del movimiento - en concreto, de la parte representada por los mensajes emitidos por Democracia Real Ya- así como los cambios y la evolución que estos han ido sufriendo a lo largo del tiempo, en tanto que discursos marcadamente atravesados por la ideología.

Partiendo del análisis de los diferentes contextos de la protesta, económico, político, social y sobre todo tecnológico, este trabajo ha demostrado cómo el movimiento $15 \mathrm{M}$, en un sentido general, fue el resultado de una compleja interacción entre los actores sociales y los contextos económicos, sociales, culturales, políticos y de comunicación que tuvieron lugar en España durante la primavera de 2011, lo que supuso la aparición de un movimiento de protesta espontáneo que, en el fondo, mostraba diferentes identidades que dificultaron tanto su identificación social como su influencia real.

En concreto, esta investigación arroja luz sobre los mecanismos utilizados para desarrollar los aspectos ideológicos en los textos. Para ello, siguiendo una metodología cualitativa se han analizado las convocatorias lanzadas por una de las plataformas más significativas el $15 \mathrm{M}$-Democracia Real Ya- en las tres grandes manifestaciones organizadas por la plataforma desde que apareciera en España en mayo de 2011.

El análisis de las llamadas a la movilización evidencia que las convocatorias de DRY se construyeron a partir de los elementos característicos del discurso ideológico y proporcionaron, por ello, descripciones autoidentitarias, descripciones de la actividad que la plataforma desarrolla como grupo, descripciones de los propósitos que desean alcanzar, descripciones de los recursos con los que cuentan (la unión de toda la ciudadanía y el espacio público: la calle) y descripciones de las normas y valores por los que se rigen como organización.

Por otro lado, nuestro estudio ha demostrado cómo los mensajes difundidos por Democracia Real Ya se articularon siguiendo la estrategia habitual de los discursos ideológicos, pues se aplica a los mensajes un esquema polarizado y maniqueo-"buenos y muchos (ciudadanía) vs. Pocos y malos" (bancos y políticos)-, en el que los significados están manipulados, estructuralmente, por el principio del favoritismo hacia el ingroup (en este caso, DRY) y la descalificación del outgroup (en este caso, 
los poderes políticos y financieros, considerados corruptos y enemigos de la plataforma).

La autorepresentación positiva de los miembros del ingroup se hizo patente discursivamente a través de diferentes mecanismos, como la configuración de un perfil victimista en la descripción de los integrantes del ingroup, la atribución de acciones positivas a los miembros de la plataforma, la fijación de propósitos y metas de carácter positivo o la asunción de valores o reglas morales y sociales de carácter virtuoso y que gozan de amplia aceptación popular.

Además, tal como hemos mostrado, el énfasis en la construcción de una imagen positiva del ingroup se hizo si cabe más evidente en las llamadas a la movilización ciudadana al combinarse con la representación negativa de los integrantes del outgroup en las tres convocatorias. En este sentido, cabe destacar que el análisis reveló cierta indefinición del outgroup, esbozado y descrito muy difusamente sobre todo en los dos primeros mensajes de DRY, por lo que el perfil antagónico de los "enemigos" de la plataforma quedó configurado sobre todo a partir de las acciones negativas que se les atribuían.

Según muestran los datos, se ha producido, asimismo, cierta evolución en la ideologización de los mensajes y en el tono empleado en las convocatorias dirigidas a la ciudadanía. Así, a pesar de que los componentes ideológicos del discurso resultan similares en los tres textos analizados, parece existir una polarización del discurso más acusada en la tercera llamada a la movilización, lo que apunta a una mayor carga política y a un mayor grado de beligerancia. El mayor nivel de crispación y radicalidad en el tercer mensaje lanzado por la plataforma se advierte, como hemos señalado, en diferentes aspectos como el empleo de mayor número de adjetivos calificativos para describir al outgroup que proyectan una imagen muy negativa de ellos o la oposición explícita del movimiento al sistema establecido.

Los resultados obtenidos a través del análisis de Leximancer permiten afirmar que el discurso de la plataforma DRY no fue unitario a la hora de dirigirse a la ciudadanía y llamarla a la movilización. En cada texto, la articulación y estructuración semántica fue distinta y el foco temático se situó en un lugar diverso. Al mismo tiempo, los datos corroboran que el discurso de la plataforma ha adoptado posiciones más extremas en su tercer mensaje a los ciudadanos, pues, en esta ocasión, el texto adopta un sesgo más ideológico y se articula en torno a los enemigos del ingroup -representados en las voces "mercados" y "sistema"- y a términos de carácter bélico y marcadamente combativos: "lucha" y "genocidio".

\section{Bibliografía}

BERRÍO PUERTA, A (2006). La perspectiva de los nuevos movimientos sociales en las obras de Sidney Tarrow, Alain Touraine y Alberto Melucci, en Estudios Políticos Núm. 29, 219-236 
CASTELlS, M. (2007). Communication, Power and Counter-power in the Network Society, in International Journal of Communication, 1, 238-266.

CASTELLS, M. (2012) \#12m15m. La Vanguardia. Disponible en: http:/www. lavanguardia.com/opinion/articulos/20120505/54289233400/manuel-castells12m15m.html. [11-08-2013].

DIANI, M. (1992). The Concept of Social Movements. The Sociological Review. Vol $40(1), 1-25$.

EGM- Estudio General de medios (2011). Audiencia de Internet- octubre-noviembre de 2011. Disponible en: http://www.aimc.es/-Audiencia-de-Internet-en-el-EGM. html. [21-08-2013].

ELTANTAWY, N., \& WIEST, J. (2011) Sep 2. The Arab Spring| Social Media in the Egyptian Revolution: Reconsidering Resource Mobilization Theory. International Journal of Communication 5:0. Disponible en: http:/www.ijoc.org/ojs/index.php/ ijoc/article/view/1242/597. [22-08-2013].

GARCÍA-JIMÉNEZ, L. (2012). E-social movements y resistencia simbólica: hacia una teoría de la comunicación y el contrapoder. Razón y Palabra, 81. Disponible en: http://www.razonypalabra.org.mx/N/N81/V81/09_Garcia_V81.pdf. [10-082013].

LARAÑA, E. (1999): La construcción de los movimientos sociales. Alianza. Madrid. MARQUÉS, J.M., MAZA, L. \& RUBIO, M. (2010). Una comparación de los ciclos inmobiliarios recientes en España, Estados Unidos y Reino Unido. Boletín Económico, Banco de España, 1, 107-119.Disponible en: http://europa.sim.ucm. es/compludoc/AA?articuloId=731124 [25-08-2013].

MORELL M. F. (2012). The Free Culture and 15M Movements in Spain: Composition, Social Networks and Synergies. Social Movement Studies, 11(3-4), 386-392.http:// economia.elpais.com/economia/2012/05/22/actualidad/1337674199_521664. html [30-08-2013].

NOGUEIRA, Ch. (2011). 580.000 personas se van de España. El País. Disponible en: http://elpais.com/diario/2011/10/08/sociedad/1318024802_850215.html [15-08-2013].

OBJOVI- Observatorio Joven de la Vivienda en España (2011). Objovi, 36. Disponible en: http://www.cje.org/es/publicaciones/publicaciones-periodicas/objovi-n-36/ [5-08-2013].

PEÑA-LÓPEZ, I., CONGOSTO, M., \& ARAGÓN, P. (2013). Spanish Indignados and the evolution of 15M: towards networked para-institutions. Big Data: Challenges and Opportunities.

PERUGORRÍA, I., \& TEJERINA, B. (2013). Politics of the encounter: Cognition, emotions, and networks in the Spanish 15M. Current Sociology, 61(4), 424-442.

TARROW, S. (2004): El poder en movimiento: los movimientos sociales, la acción colectiva y la politica. Alianza. Madrid.

TOURAINE, A. (2006). Los movimientos sociales. Revista Colombiana de Sociología. $\mathrm{N}^{\mathrm{o}} 27,255-278$.

VAN DIJK, T.A. (1996). Análisis del discurso ideológico. Traducción: Ramón Alvarado. Revisión: Teresa Carbó, en Versión, Vol. 6, 15-43. Disponible en: http:// 
segundaslenguaseinmigracion.com/L2ycomptext/Anlisisideolgico.pdf. 2013].

(2005). Discurso, conocimiento e ideología. Reformulación de viejas cuestiones y propuesta de algunas soluciones nuevas, en Cuadernos de Información y Comunicación, Vol. 10, 285-318.

(2008). Semántica del discurso, en Discurso \& Sociedad, Vol 2(1), 201-261.

ZAMORA, R. y GARCÍA, L. (2013). "New Communicative Symbolic Counterpower as a consequence of Political Distrust: An Analysis of the Discourses Around 'Occupy the Street' in Spain" paper presentado en conference regional ICA celebrada en Málaga (julio, 2013).

\section{Notas}

1 Este trabajo se enmarca en el proyecto de investigación "Análisis de los puntos de contacto más eficaces para la creación de la marca política en el público joven”, financiado por la Fundación Séneca (Murcia).

2 Desde 1986 hasta el estallido de la crisis en el año 2008, España registró un período de bonanza económica (con algunas excepciones, como la crisis de 1993) que se prolonga hasta 2008. Además de la crisis económica mundial, España sufrió a partir de ese año una crisis mayor en parte debida a una importante presencia del sector de la construcción y las elevadas deudas que las familias, las empresas y los gobiernos adquirieron debido a las bajas tasas de interés en préstamos hasta el año 2005 (Marqués, Maza y Rubio, 2010 ). La especulación en la vivienda y el alto riesgo de las hipotecas causaron una sobrevaloración de las propiedades que resultó aún más evidente con la caída de una sociedad financiera global como Leman Brothers en 2007, que puso fin a esta sobrevaloración y fue un hito en la crisis económica mundial y europea. En España, la burbuja inmobiliaria y el fuerte aumento de las tasas de interés provocó una recesión económica muy dura y sin precedentes, que fueron denunciadas por manifestantes del 15M.

3 Desde 1986 hasta el estallido de la crisis en el año 2008, España registró un período de bonanza económica (con algunas excepciones, como la crisis de 1993) que se prolonga hasta 2008. Además de la crisis económica mundial, España sufrió a partir de ese año una crisis mayor en parte debida a una importante presencia del sector de la construcción y las elevadas deudas que las familias, las empresas y los gobiernos adquirieron debido a las bajas tasas de interés en préstamos hasta el año 2005 (Marqués, Maza y Rubio, 2010 ). La especulación en la vivienda y el alto riesgo de las hipotecas causaron una sobrevaloración de las propiedades que resultó aún más evidente con la caída de una sociedad financiera global como Leman Brothers en 2007, que puso fin a esta sobrevaloración y fue un hito en la crisis económica mundial y europea. En España, la burbuja inmobiliaria y el fuerte aumento de las tasas de interés provocó una recesión económica muy dura y sin precedentes, que fueron denunciadas por manifestantes del $15 \mathrm{M}$.

4 El barómetro elaborado por el CIS en abril de 2011 mostró que los ciudadanos españoles se vieron afectados principalmente por tres problemas graves: el desempleo, la situación económica y el sistema político/partidos políticos (CIS, 2011: 3). 


\section{Las autoras}

Celia Berná Sicilia es Doctora Europea en Filología Hispánica por la Universidad de Murcia y Licenciada en Periodismo por la Universidad de Murcia. Sus ámbitos de interés investigador se centran, además de en el estudio de la incidencia que las interacciones entre léxico, sintaxis y semántica pueden tener el desarrollo de la competencia comunicativa, en el análisis de los factores que inciden en la comunicación en distintos tipos de discurso. Ha desarrollado su actividad docente e investigadora en distintos centros universitarios nacionales e internacionales, como la Universidad de La Sorbona (París IV), la Universidad de Zúrich (Suiza), la Universidad de Leipzig (Alemania) y la Universidad a Distancia de Madrid (UDIMA). Actualmente, es profesora en la Universidad Católica San Antonio de Murcia en las titulaciones de Comunicación, Turismo y Educación Infantil y Primaria.

Helena Martínez Martínez es Doctoranda en Periodismo por la Universidad Católica San Antonio de Murcia, Master en Dirección de Comunicación y Licenciada en Periodismo. Sus ámbitos de interés investigador se centran en los movimientos sociales, identidad, discurso y comunicación corporativa, branding y social media. Desarrolla su actividad investigadora en la Facultad de Ciencias Sociales y de la Comunicación de la Universidad Católica San Antonio de Murcia.

Rocío Zamora Medina es Doctora en Comunicación Pública por la Universidad de Navarra. Actualmente, trabaja como Profesora Contratada Doctora en la Universidad de Murcia, donde desarrolla tareas docentes e investigadoras en el área de los Fundamentos de la Comunicación y de la Información y en Opinión Pública y Comunicación Política. Sus líneas de investigación tienen que ver con el análisis estratégico y de recepción de mensajes aplicado al ámbito de la comunicación pública en los contextos políticos y electorales. Cuenta con numerosas publicaciones internacionales, además de textos propios en este ámbito. Ha desarrollado su proyección internacional como docente e investigadora en diferentes universidades internacionales (U. Missouri y Benedictine University of Chicago en USA) y Europeas (London School of Economics (UK), U. Wroclaw (Polonia), U. Coimbra (Portugal), U. Lumsa (Roma), U. Etna Kore (Sicilia), U. Sassari (Cerdeña), U. Turku (Finlandia), además de otras nacionales en la que ha impartido docencia en grado y posgrado (UCAM (Murcia), Universidad de Navarra, Universidad de Cadiz y Universidad de Málaga). 